\title{
A Fully Automatic CAD-CTC System Based on Curvature Analysis for Standard and Low-Dose CT Data
}

\author{
Tarik A. Chowdhury*, Paul F. Whelan, Senior Member, IEEE, and Ovidiu Ghita
}

\begin{abstract}
Computed tomography colonography (CTC) is a rapidly evolving noninvasive medical investigation that is viewed by radiologists as a potential screening technique for the detection of colorectal polyps. Due to the technical advances in CT system design, the volume of data required to be processed by radiologists has increased significantly, and as a consequence the manual analysis of this information has become an increasingly time consuming process whose results can be affected by interand intrauser variability. The aim of this paper is to detail the implementation of a fully integrated CAD-CTC system that is able to robustly identify the clinically significant polyps in the CT data. The CAD-CTC system described in this paper is a multistage implementation whose main system components are: 1) automatic colon segmentation; 2) candidate surface extraction; 3) feature extraction; and 4) classification. Our CAD-CTC system performs at $100 \%$ sensitivity for polyps larger than $10 \mathrm{~mm}, \mathbf{9 2 \%}$ sensitivity for polyps in the range 5 to $10 \mathrm{~mm}$, and $57.14 \%$ sensitivity for polyps smaller than $5 \mathrm{~mm}$ with an average of 3.38 false positives per dataset. The developed system has been evaluated on synthetic and real patient $\mathrm{CT}$ data acquired with standard and low-dose radiation levels.
\end{abstract}

Index Terms-Classification, computed tomography (CT), convexity test, feature extraction, low-dose computed tomography colonography (CTC), polyp detection.

\section{INTRODUCTION}

C OLON cancer is the leading cause of cancer-related deaths in the developed nations [19], [21]. Colon cancer statistics indicate that in the U.S. 130200 new cases were diagnosed in 2000 with 56300 people dying as a result [2]. In the U.K., 35600 new cases were identified in 1999 with colon cancer being the main contributor for 16170 deaths in 2001 [3]. In Ireland 2720 patients died due to colon cancer during the period 1998-2000 and the Irish medical statistics indicate that the colon cancer is the second leading cause of cancer-related deaths for women and the third leading cause of cancer induced

\footnotetext{
Manuscript received April 6, 2006; revised January 26, 2007. This work was supported by the Science Foundation Ireland under an Investigator Programme Grant 02/IN1/1056 and by Enterprise Ireland under a Commercialization Grant $\mathrm{CP} / 06 / 202$. Asterisk indicates corresponding author.

*T. A. Chowdhury was with the Vision Systems Group, School of Electronic Engineering, Dublin City University, Dublin 9, Ireland. He is now with the Computer Science and Engineering Department, BRAC University, 66 Mohakhali, Dhaka 1212, Bangladesh (e-mail: tachowdhury@ bracuniversity.ac.bd).

P. F. Whelan and O. Ghita are with the Vision Systems Group, School of Electronic Engineering, Dublin City University, Dublin 9, Ireland (e-mail: whelanp@eeng.dcu.ie; ghitao@eeng.dcu.ie).

Color versions of one or more of the figures in this paper are available online at http://ieeexplore.ieee.org.

Digital Object Identifier 10.1109/TBME.2007.909506
}

deaths for men [19]. Typically, colon cancer develops as an intestinal polyp which is an abnormal growth of the colon tissue and in time the colorectal polyps may become cancerous. The process of polyp growth is slow (it may take few years until the polyps become cancerous) and the early detection and removal of polyps via screening has proven to be an effective procedure to reduce the colon cancer mortality [4], [13].

The most common methods used in identifying the presence of colorectal polyps in a clinical setting are the faecal occult blood test (FOBT), flexible sigmoidoscopy, barium enema, DNA stool test, and optical colonoscopy. From the aforementioned methods, colonoscopy is widely accepted as the screening technique that returns the highest sensitivity in polyp detection [29]. The colonoscopy examination is performed by the gastroenterologists by using a flexible colonoscope to investigate the inner wall of the colon. The colonoscope is fitted with a miniature camera and light unit to allow the gastroenterologist to evaluate any abnormal changes on the colon tissue. If abnormal growths on the colon are discovered, the gastroenterologist can use the channels of the colonoscope to preserve biopsies (that can be used for later examination) or even remove the colorectal polyps if this is necessary. Prior to the scheduled colonoscopy investigation, the patient is instructed to follow a strict diet for $24 \mathrm{~h}$ in order to perform bowel cleansing and typically the colonoscopy examination takes between 20-60 min while the patient is sedated. The main disadvantage associated with the optical colonoscopy is the invasive nature of the procedure, although the risk of bowel perforation by the colonoscope is small $(0.005 \%$ of the total number of investigated cases). For $5 \%$ of the patients the colonoscope is not able to reach the caecum point, which results in an incomplete colon examination [22].

Computed tomography colonography (CTC) is a minimally invasive technique that is considered as an alternative for optical colonoscopy for the detection of colorectal polyps [6], [10], [13], [29]. The CTC method involves the computed tomography (CT) scan of the patient's abdomen and the resulting data is evaluated by the radiologists to detect whether the colorectal polyps are present by analyzing either axial CT images or the 3-D surface of the air insufflated colon (during the CTC examination, the colon is insufflated with air at the maximum level tolerated by the patient in order to obtain adequate colon distention). The CTC examination is significantly less invasive than the standard optical colonoscopy, and recent studies indicate that sensitivity in polyp detection achieved by CTC is comparable to the sensitivity offered by the standard approach [6], [29]. With the introduction of the multislice $\mathrm{CT}$ scanners, the amount of data to be examined by the radiologists has increased dramatically and 
a dataset can consist of 200 to 500 axial images depending on the patient height and the resolution of the CT data. Thus, the visual examination of such a large amount of data is a time consuming procedure, and the results are not always reproducible since they are often affected by eye fatigue and the subjectivity of the human operator [7]. The development of computer-aided detection (CAD) techniques has led to the introduction of computer-based automated systems that can generate reproducible results with a high sensitivity in detection of clinically significant polyps (i.e., larger than $5 \mathrm{~mm}$ ) [12], [14], [17], [25], [31]. The main problem with the current range of CAD-CTC systems is the high number of false positives [18] that are generated (mainly by convex colon structures that mimic the shape of the polyps such as the haustral folds, residual faecal material, inflation tube, ileocecal valve, etc). A substantial discussion on the factors that generate the false positives can be found in Yoshida et al. [32] where they addressed the main issues that the CAD-CTC systems have to confront in order to reduce the level of false positives to a level that would make their use feasible in clinical examinations.

There are a large number of approaches that have been developed to perform robust polyp identification in CT data. Most of the developed systems use the geometrical assumption that the nominal shape for polyps is spherical/ellipsoidal whereas the folds resemble more elongated/cylindrical shapes [14], [25], [26], [30]. Thus, these systems attempt to exploit the geometrical differences between these two main categories of colon features that are represented by polyps and folds. The problem of polyp identification is a complex task since often the difference in shape between polyps and folds is very subtle. In addition they may have differing size and shape when compared to the nominal model used to characterize the main colon features.

The aim of this paper is to detail the full implementation of a CAD-CTC system that has been developed as a modular system where all components are interconnected using a systems engineering approach. Section II details the overall structure of the developed CAD-CTC system. Section III discusses the colon segmentation algorithm. Section IV introduces the candidate surface extraction algorithm and the convexity test that is applied to remove the voxels with low convex characteristics. Section $V$ presents the feature extraction and the associated classification scheme required for robust polyp identification. Section VI discusses the experimental results while Section VII presents a performance characterization of our system and other publicly documented CAD-CTC applications. Section VIII concludes this paper.

\section{OVERVIEW OF THE DEVELOPED CAD-CTC SYSTEM}

The main computational components of our CAD-CTC system are illustrated in Fig. 1. This involves decoding the CT data [35], [36], 3-D bilinear interpolation (to produce an isometric dataset), colon segmentation, the extraction of candidate surfaces, and classification. In this regard, the colon segmentation stage may appear to be a trivial task (e.g., using simple region growing algorithm) since there is a step voxel intensity difference between the air-filled areas $[-1000$ Hounsfield unit (HU)] and the colon tissue (40 HU). Unfortunately, blockages that are caused by water left in the colon or by residual faecal material can occur resulting in a partial segmentation of the

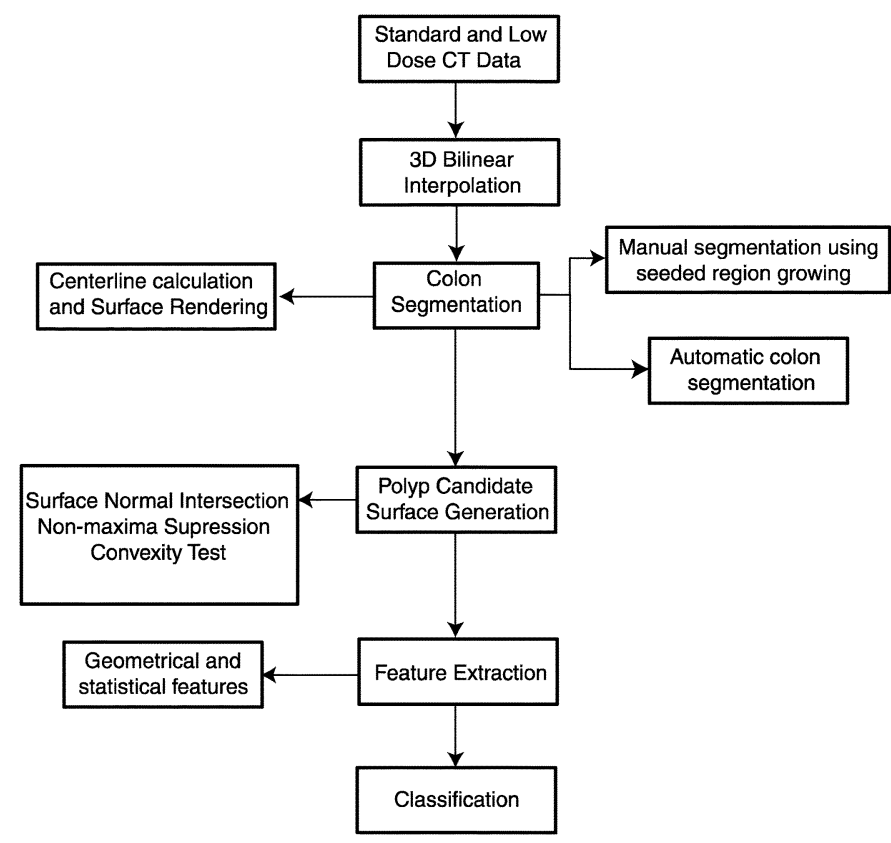

Fig. 1. Overview of the developed CAD-CTC system.

colon. Thus, automatic colon segmentation is far from a trivial task, requiring the investigation of a more involved approach.

The second main element of the CAD-CTC system involves the extraction of the polyp candidate surfaces by evaluating the local convexity of the colon surface as sampled by the distribution of the normal intersections. The third and the most sophisticated computational component of the system is represented by the feature extraction and classification scheme that is employed to extract the most robust features that can be used for polyp identification. The developed CAD-CTC system has other computational strands that perform the colon subsegmentation and the centerline calculation [23] that aid the 3-D colon navigation and the 3-D visualization of the polyps detected by the system.

\section{Automatic Colon Segmentation}

The segmentation of well-distended colons in CT data is a relatively simple task since there is a high voxel difference between the gas and colon tissue interface (in the case of a fully distended colon this can be done using a seeded region growing algorithm). If the colon data is collapsed (due to the presence of blockages caused by the residual faecal material and/or water) then region growing can only be applied in a semiautomated way which requires the manual selection of the multiple seed points. Our aim is to devise a technique that is able to identify the colon in CT data without any user intervention. The automation of the colon segmentation process has to address two main problems. The first problem is represented by the obvious fact that the colon is not the only air-filled organ in CT data. Other air-filled organs include the lungs, stomach, and small intestines and the air-filled regions associated with them in the CT data have to be identified and discarded in order to segment the colon. The second major problem is the achievement of the complete colon segmentation even in cases where the residual water and faecal material create multiple collapses in the colon 


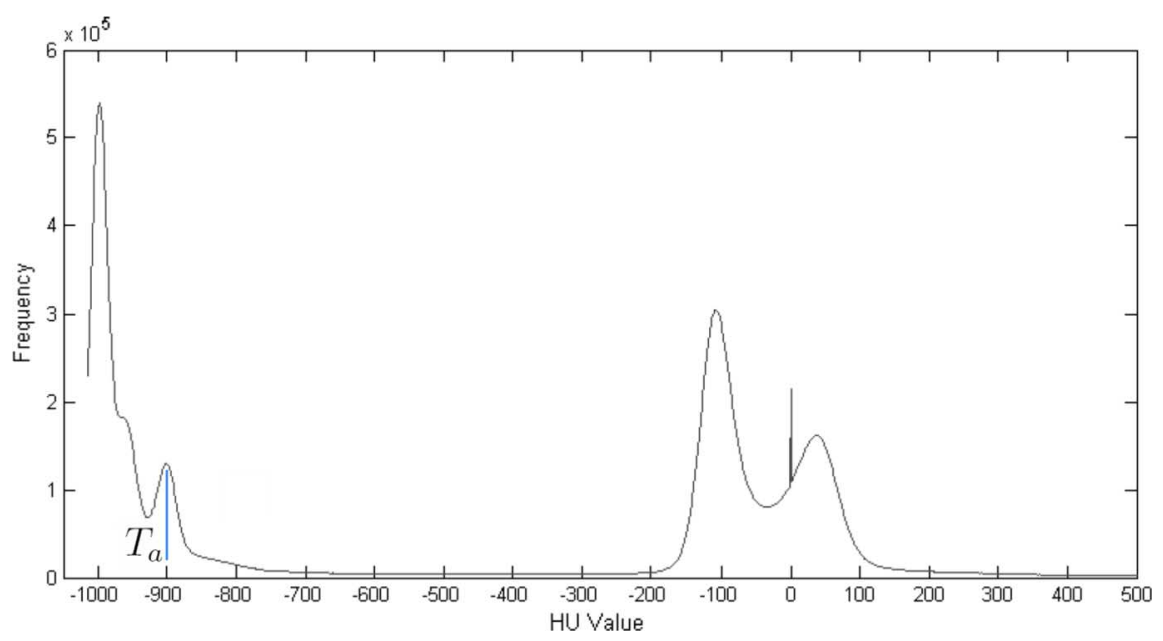

Fig. 2. Histogram-based selection of the threshold $\left(T_{a}\right)$.

data. In order to address these issues we have developed a multistage colon segmentation process that includes the removal of air region around the body, lung segmentation, and colon reconstruction using anatomical knowledge and volume/length $(\mathrm{V} / \mathrm{L})$ analysis.

\section{A. Surrounding Air Voxel Removal}

The first stage in the colon segmentation algorithm is the removal of the air around the body, which is performed by using a standard seeded region growing algorithm. The seed point for the region growing is selected as the top left voxel of the first slice of the CT dataset. The threshold value that evaluates the similarity between the seed point and the neighboring voxels during the region growing process is automatically selected as the second peak $\left(T_{a}\right)$ of the global histogram of the dataset. (Typically the $T_{a}$ threshold is situated close to the $-900 \mathrm{HU}$ point as illustrated in Fig. 2.)

\section{B. Lung Detection}

Next, the algorithm performs the removal of the lungs from the CT data. In the head-first supine or prone CT data the lungs are always present in the first slice. After the removal of the region defined by the air around the patient body, the algorithm initiates the region growing process for all air-filled regions where the seeds are the voxels with a HU value lower then the threshold $T_{a}$ (Fig. 2). The algorithm also checks for the presence of multiple blood vessels and if detected the region is defined as being part of the lungs and eliminated from the CT data as illustrated in Fig. 3.

\section{Colon Segmentation}

After the removal of the air voxels (from around the patient) and the lung tissue, the only air-filled areas left in the CT dataset are the small intestines, stomach, and the colon. The algorithm starts to label the remaining air-filled regions using a reconstruction by dilation algorithm. The labelling of the air-filled regions is performed in two stages. In the first phase any of the air voxels below the threshold $T_{a}$ are the seeds for the region growing algorithm, and the process is iteratively applied until all connected voxels are labelled and the algorithm calculates the endpoints for each labelled region.
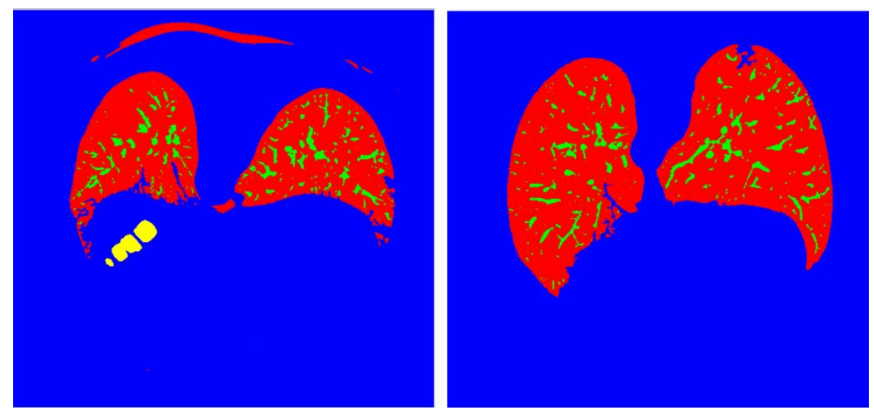

Fig. 3. Identification of the lung tissues. Note that the colon segments situated on the left image (yellow/light) are not assigned as lung tissue as they do not have any blood vessels (green/dark).

In order to identify the colon in the labelled data, we calculate the volume and the geodesic distance (length) between the endpoints (using the shortest path Dijkstra algorithm [5]) for each air-filled region. The volume/length (V/L) measure is calculated for each air-filled region, and this measure is used as the primary discriminative feature to differentiate the colon segments from other segments associated with the small intestines. Fig. 4 illustrates the performance of this shape index when the $\mathrm{V} / \mathrm{L}$ values are plotted for 35 datasets. It can be observed that the $\mathrm{V} / \mathrm{L}$ value for well-distended colon parts is greater than $600 \mathrm{~mm}^{2}$ and to allow a high degree of tolerance we have experimentally determined that the well-distended colon segments should have a V/L value greater than $300 \mathrm{~mm}^{2}$. This $\mathrm{V} / \mathrm{L}$ threshold is suitable to robustly identify the large colon segments, but it is not efficient when discriminating the small colon parts situated between large colon segments and parts of the small intestines. In order to recover these small segments we need to employ anatomical knowledge by enforcing an ideal model of a well-distended colon (see Fig. 5) during the colon reconstruction process.

The algorithm attempts to reconstruct the colon starting with the labelled segment placed close to the rectum using the knowledge that the colon is the only air-filled region at that position in the CT data. For a fully distended colon the region detected near to the rectum position will have one end-point close to the rectum and the other close to the caecum. To declare a colon 


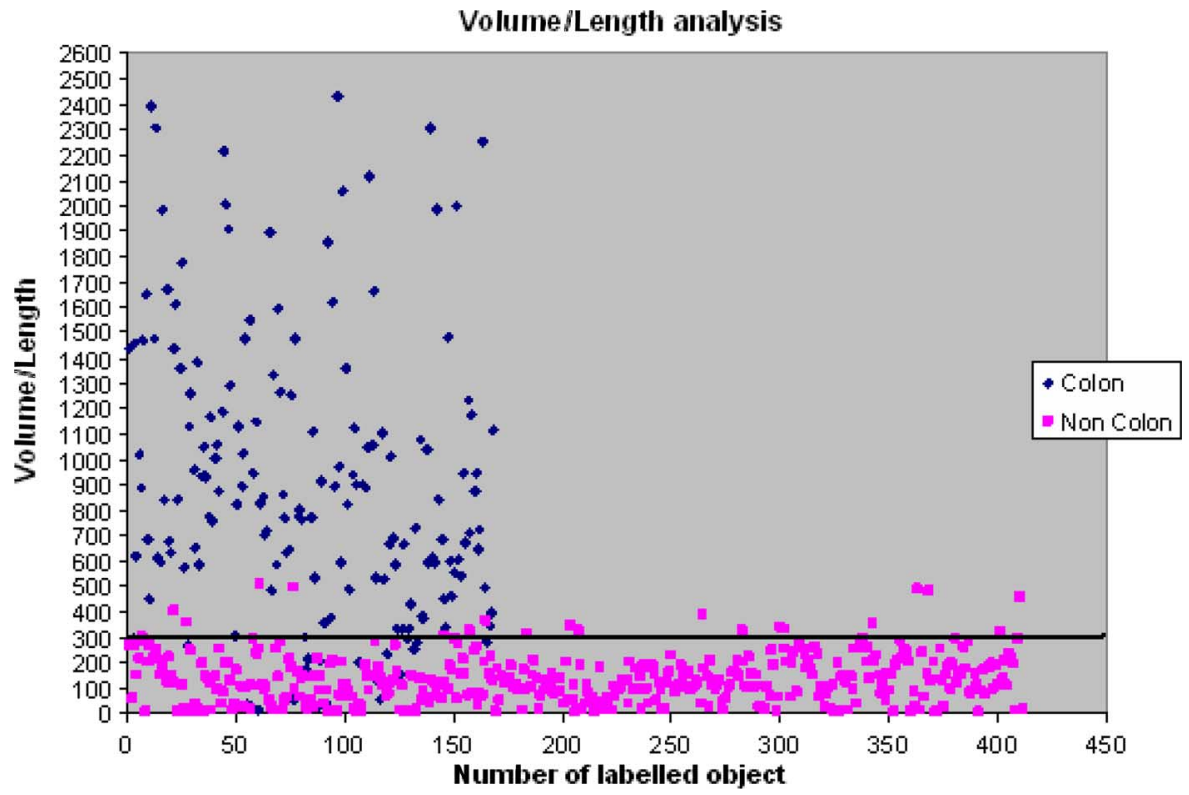

Fig. 4. V/L shape index for colon (blue/dark) and noncolon (red/light) air-filled segments.

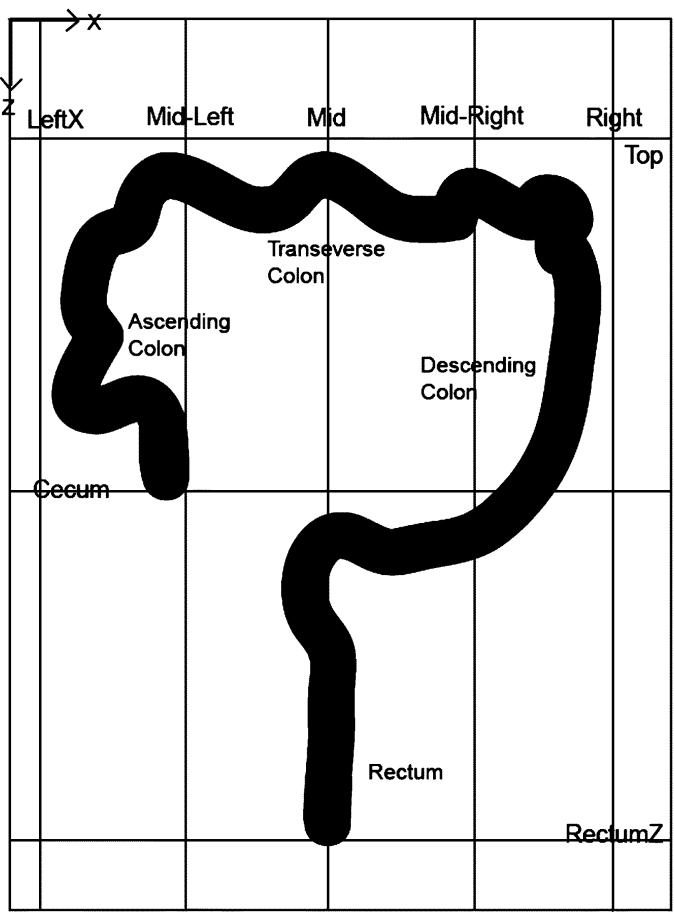

Fig. 5. Ideal geometrical model for a well-distended colon.

fully distended it must fulfil the ascending and descending geometry in accordance with the ideal colon model depicted in Fig. 5. If the colon does not meet these geometrical constraints it is declared as a collapsed colon.

The reconstruction of the colon lumen in the collapsed CT data is performed in two steps. Initially the large segments with $\mathrm{V} / \mathrm{L}$ values greater than $300 \mathrm{~mm}^{2}$ are detected. The colon reconstruction starts with the segment located closer to the rectum, and the Euclidean distance between the closest endpoints of adjacent candidate segments is calculated. These large candidate segments are identified as part of the colon if their orientations match the geometric constraints imposed by the anatomical model of an ideal colon (Fig. 5). Once this process is completed, the algorithm initiates the second phase of the colon reconstruction process by analyzing the small segments that can be either parts of the colon or parts of the small intestines. The inclusion of the small segments is evaluated based on the orientation of the centerline using the approach detailed in [23]. In order to eliminate the high-frequency components, the centerline has been smoothed and down-sampled by a factor of seven and the resulting points are interpolated using B-splines. The small labelled segments $\left(\mathrm{V} / \mathrm{L}<300 \mathrm{~mm}^{2}\right)$ are declared as part of the colon if they fulfil a distance threshold and the angular constraint imposed by the orientation of the centerline. (The curvature of the centerline is sampled by its local gradient. The local gradient of the interpolated centerline has higher values for candidate segments generated by the small intestines than the gradient values associated with colon parts.)

Fig. 6 illustrates the segmentation of four collapsed colons performed by the radiologists from Mater Misericordiae Hospital (Ireland) and the segmentation obtained after the application of our automated colon segmentation method. The processing time required by the developed colon segmentation algorithm when executed on a 1.6-GHz Pentium IV personal computer with 1 GB RAM to process one standard dataset is depicted in Table I. The average size of a bilinear interpolated (isometric) CT dataset is $300 \mathrm{MB}$.

\section{CANDidate Surface EXTRACTION}

The second main component of the system is represented by the extraction of the polyp candidate surfaces using an enhanced version of the technique detailed in Kiss et al. [14]. The developed method evaluates the intersection of the normal vectors for a number of normal concentration $(N C)$ points (in the original implementation developed by Kiss et al. [14] the 


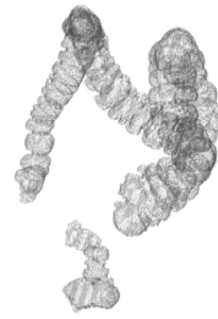

(a)

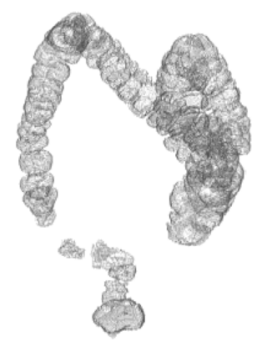

(e)

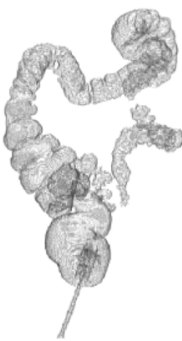

(b)

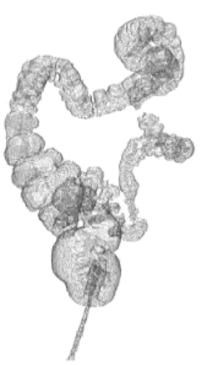

(f)

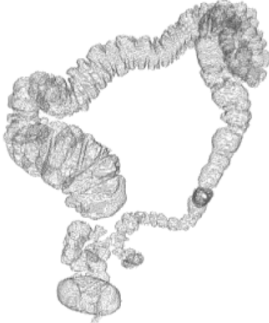

(c)

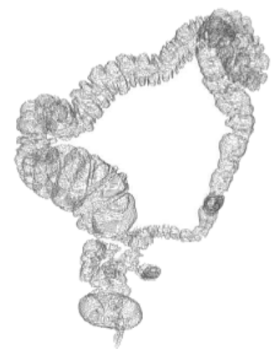

(g)

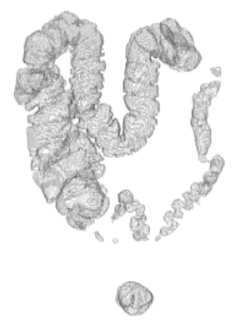

(d)

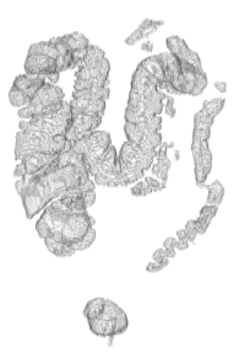

(h)

Fig. 6. Manual and automated segmentation of four collapsed colons. (a)-(d) Colon segmentation performed by the radiologists from Mater Hospital. (e)-(h) Colon reconstruction performed by our colon segmentation algorithm.

TABLE I

Processing Time ReQuired to Process One Standard CT Dataset

\begin{tabular}{|c|c|}
\hline $\begin{array}{c}\text { Component of the colon segmentation } \\
\text { algorithm }\end{array}$ & $\begin{array}{c}\text { Processing time } \\
\text { (seconds) }\end{array}$ \\
\hline Surrounding air voxel removal & 28.65 \\
\hline Lung detection & 13.03 \\
\hline Labelling & 128.32 \\
\hline V/L analysis & 19.63 \\
\hline Centerline calculation & 15.74 \\
\hline Overall processing time & 205.37 \\
\hline
\end{tabular}

normal concentration (NC) points are referred to as Hough points (HP) since the construction of the histograms that record the intersections of the normal vectors mimics the action used in a Hough transform accumulator array) that are uniformly distributed from 2.5 to $10 \mathrm{~mm}$ in the normal direction as indicated in Fig. 7.

The uniform distribution of these seven $\mathrm{NC}$ points along the normal orientation is justified by the fact that the CT dataset is first subjected to a 3-D bilinear interpolation that is applied in order to obtain an isometric dataset (the voxel resolution is approximately the same in all directions). This facilitates the application of the same colon segmentation and polyp detection techniques to datasets that are acquired with different scanning protocols (additional details about data acquisition protocols are provided in Section VII).

To evaluate the intersections between the normal vectors we have constructed a 3-D histogram for each point in the NC points set. To eliminate the noise in the 3-D histogram we have smoothed the 3-D data using the Gaussian weighted averaging operation. As the Gaussian operator is separable, this involves a

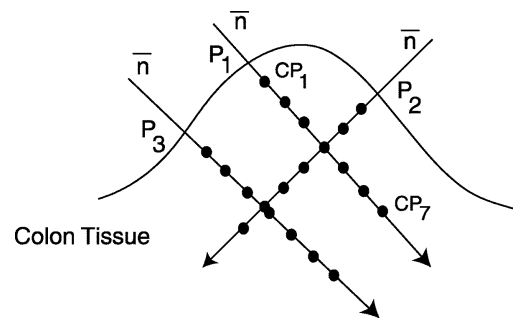

Fig. 7. Surface normal intersection and the distribution of the NC points in the normal direction for each voxel of the colon wall.

convolution of the CT data with 1-D filters in the $x, y, z$ directions as illustrated in (1).

$$
\begin{aligned}
w(x, y, z)= & w(x) * w(y) * w(z), \quad \text { where } \\
w(k)= & \frac{1}{\sqrt{2 \pi} \sigma} e^{\frac{-k^{2}}{2 \sigma^{2}}} \text { and } * \text { is the convolution operator } \\
V_{\text {smooth }}(x, y, z)= & \sum_{i=-m}^{m} \sum_{j=-m}^{m} \sum_{k=-m}^{m} v(x+i, y+j, z+k) \\
& \times w(i, j, k)
\end{aligned}
$$

where the standard deviation $\sigma=1.0$ and the half size of the 1 -D Gaussian filter $m=5$. The NC points that have more than four intersections generate the initial centers of the candidate surfaces. Since the polyps and folds may have irregular shapes, a convex surface may generate multiple center points and this will result in a high fragmentation of the convex surface.

To eliminate this problem we have developed a nonmaxima suppression algorithm based on an exhaustive search within the distance of $10 \mathrm{~mm}$ from the point with the local maximum number of intersections. The final surface is generated by all 


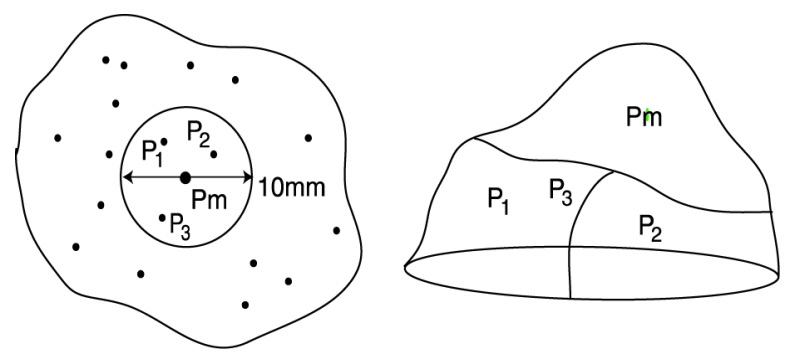

Fig. 8. Nonmaxima suppression procedure. (a) The distribution of the NC points within a sphere with a diameter of $10 \mathrm{~mm}$ centerd on the NC point $\left(P_{m}\right)$ that has the maximum number of intersections. (b) The surface generated by the voxels that are associated with NC points with more than four intersections that are situated inside the $10-\mathrm{mm}$ search space.

voxels that have $\mathrm{NC}$ points with more than four intersections within the investigated search space (see Fig. 8). The application of the nonmaxima suppression produces continuous convex surfaces but may also include points with weak or nonconvex characteristics that are situated at the periphery of the extracted surface. To remove these undesired surface points we developed a simple procedure, referred to as the Surface Convexity Test, which evaluates the convexity of all voxels that define the candidate surface.

\section{A. Surface Convexity Test}

In order to remove the nonconvex voxels from the candidate surface we need to recalculate the center of the surface. The center of the candidate surface is the NC point that has the highest Gaussian distribution and is calculated using the following expression:

$$
G m_{i}=\sum_{j=1}^{N} e^{\frac{-x_{i j}^{2}}{2 \sigma^{2}}}
$$

SurfaceCenter $=N C P\left(\arg \max _{i}\left(G m_{i}\right)\right), \quad i=1, \ldots, N$

where $x_{i j}$ is the distance between the NC points with indexes $i$ and $j, \sigma$ is the standard deviation and is set to 1.0 , and $N$ is the number of $\mathrm{NC}$ points $(\mathrm{NCP})$ that generate the surface. Once the center of the surface has been determined as illustrated in Fig. 9 we can evaluate the convexity of each surface point using the following test.

Let $S$ be a surface voxel and $\bar{n}$ be the normal vector at the surface voxel $S . Q$ is the intersection point between the surface normal and the perpendicular line from the center of the cluster to the surface normal as illustrated in Fig. 10. As can be observed in Fig. 10, the scalar product between the oriented vector $(\overline{Q S})$ and normal vector $\bar{n}$ indicates precisely whether the point is convex or not. If the scalar product $\overline{Q S} \bullet \bar{n}>0$ (vector $\overline{Q S}$ and the normal vector $\bar{n}$ have the same orientation), the surface point is considered to be nonconvex, and it will be removed from the candidate surface. In Fig. 10 it can be observed that the points $s_{1}$ and $s_{4}$ will be removed from the candidate surface, as they do not obey the convexity test. After the removal of the nonconvex surface voxels, the algorithm starts to evaluate the surface continuity. If the algorithm finds discontinuities in the candidate surface it is assumed that the surface is generated by more than one convex colon feature and the algorithm divides
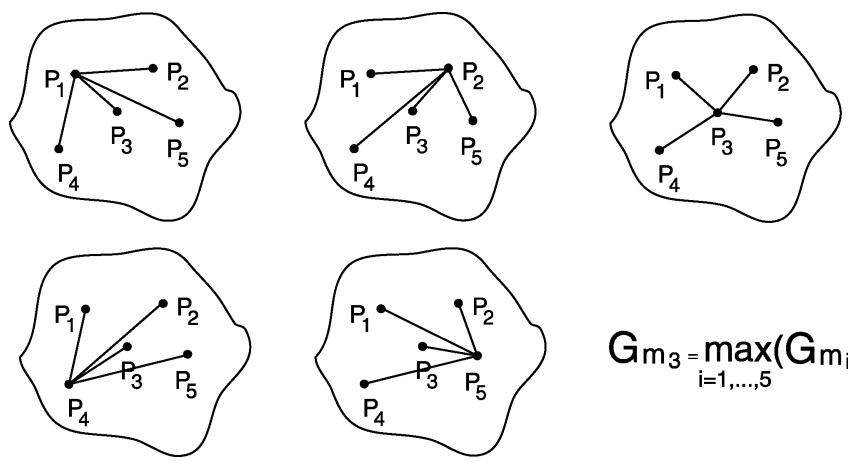

$\mathrm{Gm}_{3}=\max _{\mathrm{i}=1, \ldots, 5}\left(\mathrm{Gm}_{\mathrm{i}}\right)$

Fig. 9. Center of the candidate surface is approximated by the NC point $\left(P_{3}\right)$ that has the highest Gaussian distribution.

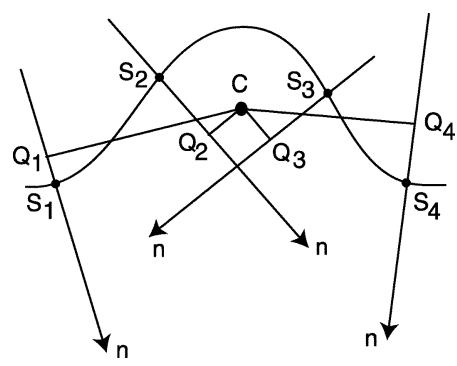

Fig. 10. Convexity test. The point $\mathrm{C}$ is the calculated center of the candidate surface. The surface points $s_{2}$ and $s_{3}$ pass the convexity test whereas the surface points $s_{1}$ and $s_{4}$ and their associated NC points are removed since do not obey the condition $\overline{Q S} \bullet \bar{n} \leq 0$.

the candidate surface until no discontinuities are encountered. If the initial candidate surface has been divided, the algorithm recalculates the center and associated radius for each new surface using the expression illustrated in (2).

\section{FEATURe EXTRACTION AND Classification}

Our objective is to extract only the features from the candidate surfaces that offer the best discrimination between polyps and folds. At this stage it is useful to recall that polyps and folds have various shapes and sizes and in some situations they can be almost indistinguishable. Thus, the discriminative features employed to perform polyp detection have to optimally exploit the geometrical difference between polyps and folds in order to achieve robust polyp detection at a low level of false positives. In general the number of features required to perform optimal classification is in direct relation to the difficulty of the problem. In this sense, a large number of features may offer more information that can be used in the classification process but their use is problematic as they may have different levels of discrimination (i.e., some features are better in discriminating large polyps from folds while other are better in discriminating medium sized polyps from folds) and as a result they will be difficult to be used in a coherent classification unless we have robust information that allows a preliminary separation between the candidate surfaces (i.e., segregation of candidate surfaces by size). Thus, the selection of the optimal features can be obtained by performing a detailed analysis of the discrimination power of each feature. Ideally they should be able to provide discrimination between all types of polyps from folds. Previous approaches to CAD-CTC (as discussed in Section VII) employ a large range of features that are used for polyp/fold classification using a committee of 


\section{Surface analysis for polyp}

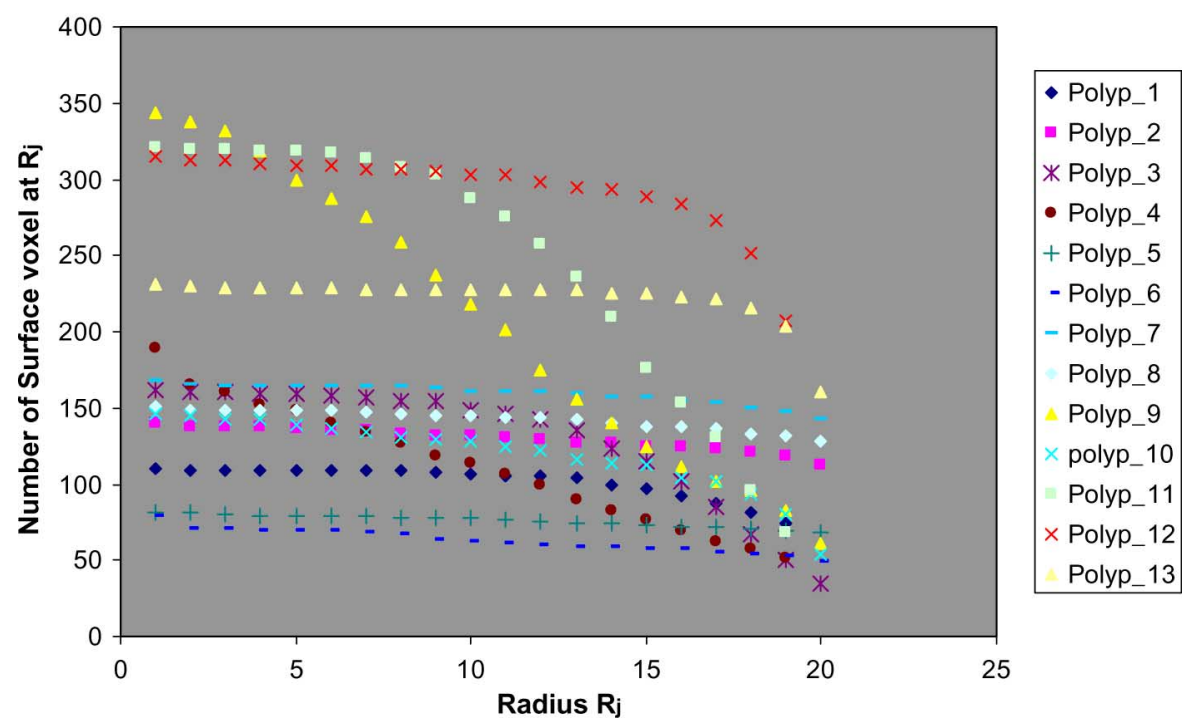

Fig. 11. Number of surface voxels at each radius $\left(R_{i}\right)$ for polyp classes (classes are sorted in ascending order with respect to the size of the polyps/folds).

classifiers where each classifier is tuned to identify a particular type of polyp (e.g., polyps with spherical shapes). As a result the features used by a particular classifier are tuned to maximize the detection of a particular polyp shape. Nonetheless this approach is successful if the features employed provide a primary separation between other categories of polyps and folds; otherwise the accuracy of the system will be affected by an inefficient voting process [12]. For our implementation we approached the classification process differently by using a relatively small feature set, and we placed the emphasis on the extraction of the most discriminative features and evaluating their performance with respect to polyp/fold discrimination based on observation, thus reducing the burden on the classification stage. Using the knowledge that the nominal model for polyps is spherical and the nominal model for folds is cylindrical, we extracted a number of key features, namely: the standard deviation (SD) of the surface variation, the SD of the three axes of the fitted ellipsoid, SD of the ellipsoid fit error, and SD of the sphere fit error and the value of the Gaussian distribution. These features generate a low-dimensional model that describes the candidate surface that is used to classify the candidate surfaces into polyps and folds.

\section{A. Standard Deviation of the Surface Variation}

The aim of this feature is to sample the rate of surface change that evaluates the distribution of the surface voxels with respect to their position from the surface center. In order to determine the standard deviation (SD) of the surface change we need to calculate the number of surface voxels that are placed at each radius starting from $d_{\max }$ (maximum Euclidean distance between the surface voxels and the center of the candidate surface) toward the minimum radius that was set experimentally to $1 \mathrm{~mm}$ (this value was chosen as the voxel resolution of a typical CT dataset is $0.7 \mathrm{~mm}$ width, $0.7 \mathrm{~mm}$ height, and $0.8 \mathrm{~mm}$ depth). Our goal is to find out how many voxels from the candidate surfaces are situated at a particular distance from the surface center, this will generate the surface number $\mathrm{SN}$. The relationships required to calculate the surface number $S N_{i}$ for each radius are illustrated in the following:

$$
\begin{aligned}
\text { Step } & =\left(d_{\max }-1\right) / N \\
R_{i} & =d_{\max }-\text { Step } * i \text { for } \quad i=1, \ldots, N \\
S N_{i} & =\sum_{R_{i}} \text { Surface }
\end{aligned}
$$

where $N$ is the number of NC points that generate the candidate surface.

Figs. 11 and 12 depict the voxel distribution with respect to each radius $R_{i}$ for different classes of polyps and folds that are ordered by size. From these diagrams it can be observed that the surface change for candidate surfaces generated by folds decrease rapidly while the rate in surface change for polyps is almost unchanged with the incremental increase in radius. Thus the surface number can be used to measure the change in curvature and this is best sampled by the standard deviation (SD) that is calculated using the following:

$$
\begin{aligned}
S N_{\text {mean }} & =\sum_{i=1}^{N} S N_{i} \\
S N_{\text {inorm }} & =\frac{S N_{i}}{S N_{\text {mean }}} \text { for } i=1, \ldots, N \\
S D & =\sqrt{\frac{1}{N} \sum_{i=1}^{N}\left(S N_{\text {inorm }}-S N_{\text {mean }}\right)^{2} .}
\end{aligned}
$$

The discrimination offered by the standard deviation of the surface variation for different classes of polyps and folds is illustrated in Table II. It can be observed that this feature is quite effective in discriminating the polyps from all types of folds. In particular we observe the robust discrimination between the 


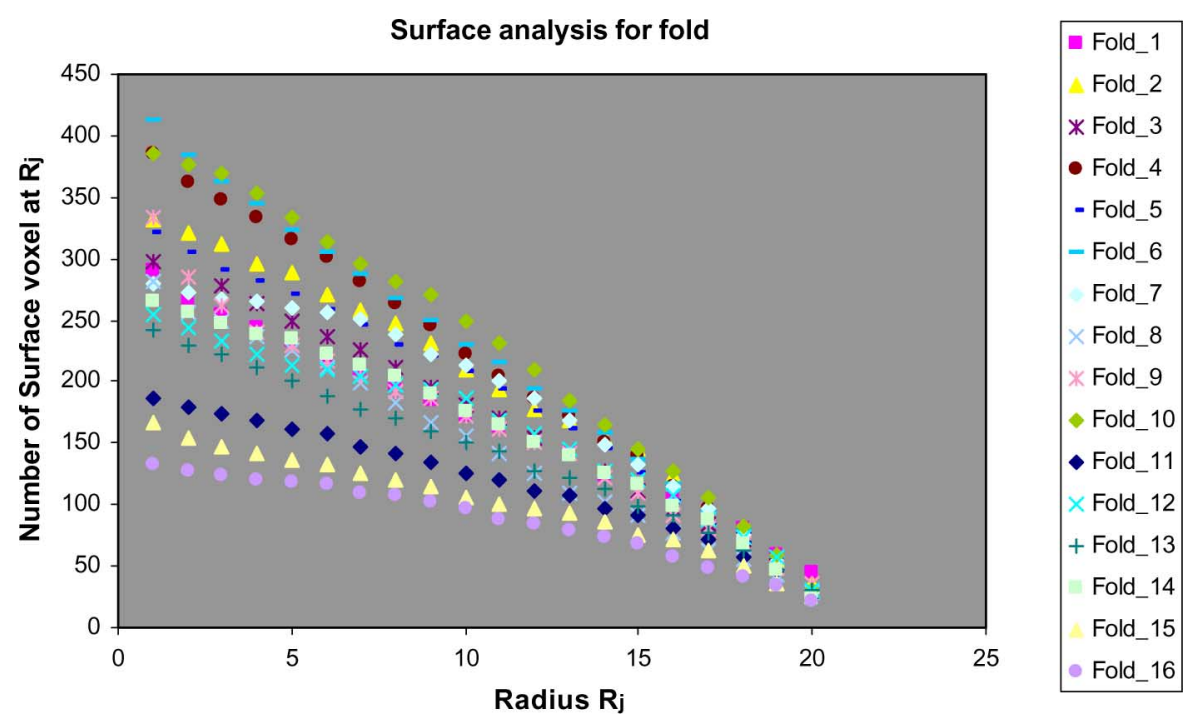

Fig. 12. Number of surface voxels at each radius $\left(R_{i}\right)$ for fold classes.

TABLE II

Class Means (CM), Class Standard Deviations (SD) of the Geometrical, and Statistical Features for PolyPs and Folds

\begin{tabular}{|c|c|c|c|c|c|c|c|c|c|}
\hline & & Polyp_1 & Polyp_2 & Polyp_3 & Polyp_4 & Fold_1 & Fold_2 & Fold_3 & Fold_4 \\
\hline \multirow{2}{*}{$\begin{array}{l}\text { SD of the surface } \\
\text { change }\end{array}$} & $\mathrm{CM}$ & 0.0938 & 0.0896 & 0.1353 & 0.2491 & 0.4747 & 0.4382 & 0.4369 & 0.3225 \\
\hline & SD & 0.0486 & 0.0432 & 0.1144 & 0.1092 & 0.0590 & 0.0475 & 0.0436 & 0.0603 \\
\hline \multirow{2}{*}{$\begin{array}{l}\text { SD of the ellipsoid } \\
\text { fit error }\end{array}$} & $\mathrm{CM}$ & 0.0433 & 0.0529 & 0.0451 & 0.0909 & 0.2346 & 0.2753 & 0.2937 & 0.2124 \\
\hline & SD & 0.0231 & 0.0357 & 0.0349 & 0.0860 & 0.1775 & 0.0964 & 0.1108 & 0.0718 \\
\hline \multirow{2}{*}{$\begin{array}{l}\text { SD of the first axis } \\
\text { of the fitted } \\
\text { ellipsoid }\end{array}$} & $\mathrm{CM}$ & 0.0838 & 0.0773 & 0.0932 & 0.1630 & 0.2457 & 0.3500 & 0.4234 & 0.3901 \\
\hline & SD & 0.0345 & 0.0387 & 0.0913 & 0.1087 & 0.1450 & 0.1404 & 0.1625 & 0.1858 \\
\hline \multirow{2}{*}{$\begin{array}{l}\text { SD of the second } \\
\text { axis of the fitted } \\
\text { ellipsoid }\end{array}$} & $\mathrm{CM}$ & 0.0744 & 0.0718 & 0.1015 & 0.1647 & 0.3586 & 0.4072 & 0.4338 & 0.3300 \\
\hline & SD & 0.0452 & 0.0602 & 0.1195 & 0.1229 & 0.1515 & 0.1368 & 0.1337 & 0.1161 \\
\hline \multirow{2}{*}{$\begin{array}{l}\text { SD of the third axis } \\
\text { of the fitted } \\
\text { ellipsoid }\end{array}$} & $\mathrm{CM}$ & 0.0773 & 0.0731 & 0.0577 & 0.1797 & 0.3752 & 0.3144 & 0.3464 & 0.2963 \\
\hline & SD & 0.0504 & 0.0460 & 0.0472 & 0.0905 & 0.1428 & 0.1345 & 0.1547 & 0.1673 \\
\hline \multirow{2}{*}{$\begin{array}{l}\text { SD of the sphere fit } \\
\text { error }\end{array}$} & $\mathrm{CM}$ & 0.0376 & 0.0369 & 0.0425 & 0.0892 & 0.1609 & 0.1796 & 0.2130 & 0.1740 \\
\hline & $\mathrm{SD}$ & 0.0155 & 0.0169 & 0.0421 & 0.0520 & 0.0715 & 0.0475 & 0.0473 & 0.0551 \\
\hline \multirow{2}{*}{$\begin{array}{l}\text { Gaussian } \\
\text { distribution }\end{array}$} & $\mathrm{CM}$ & 69.3193 & 158.4126 & 269.5211 & 320.6261 & 72.9243 & 68.7634 & 50.5330 & 45.3656 \\
\hline & $\mathrm{SD}$ & 13.4993 & 65.9292 & 104.7388 & 200.3946 & 15.2979 & 11.8311 & 6.8906 & 7.5972 \\
\hline
\end{tabular}

candidate surfaces generated by folds and those generated by large polyps $(>10 \mathrm{~mm})$.

\section{B. Statistical Features}

To complement the SD of the surface variation calculated using (8), we calculate additional features to improve the discrimination between polyps and folds by evaluating the shape difference between polyps and folds. Since the nominal model for polyps is ellipsoidal/spherical and the nominal model for folds is cylindrical, we calculate a number of statistical features that evaluate the deviation from the spherical/ellipsoidal model for colon candidate surfaces in the least squares sense [14], [16]. In this regard, we calculate the ellipsoid fitting for each surface generated using (5), and we calculate the SD of the fit error. The discrimination offered by this statistical feature is illustrated in Table II.
Additional statistical features are calculated to further describe the differences between ellipsoidal and cylindrical shapes and this involves calculating the SD of the variation of the each axis of the fitted ellipsoid. The discrimination offered by these features can be observed in Table II (note that these statistical features offer the best discrimination between the small/medium polyps and all types of folds). In a similar fashion we have calculated the SD of the sphere fitting error for each cluster surface $S N_{i}$ and the discrimination in polyp identification offered by this feature is illustrated in Table II. It can be observed that this feature is robust in discriminating all types of polyps from folds.

The last feature examined is the maximum value of the Gaussian distribution that is calculated for each cluster surface $S N_{i}$ and its performance in polyp/fold discrimination is depicted in Table II. The maximum value of the Gaussian distribution feature allows efficient separation between the large polyps $(>10 \mathrm{~mm})$ and folds. The discrimination offered by this feature is particularly useful as the previous features outlined 
while able to robustly discriminate small/medium polyps from folds, are less robust when dealing with discrimination between larger polyps and folds.

Table II illustrates the class means for a number of representative polyp and fold surfaces that were used to train the classifiers. These polyp and fold samples were segregated by size in ascending order by evaluating the number of voxels that define the surface and the Gaussian value of the surface center [as calculated using (2)].

\section{Classification}

In developing a suitably robust classification scheme we have analyzed the performance of two different classifiers to determine their contribution to the reduction of false positives, a key objective of any CAD-CTC classification stage. These are the feature normalized nearest neighbor (FNNN) classifier [8] and the probabilistic neural network (PNN) classifier. The FNNN classification scheme consists of two standard stages. Firstly, the training database is created using the features detailed in the previous section for each class of polyps and folds. The features of each class were normalized in order to avoid the situations where the features with largest values overpower the remaining ones. The feature normalization scheme has been applied to normalize each feature to zero mean and unity variance as follows:

$$
\begin{aligned}
m_{i} & =\frac{\sum_{j=1}^{k} x_{j}[i]}{k} \quad s_{i}=\sqrt{\frac{\sum_{j=1}^{k}\left(x_{j}[i]-m_{i}\right)^{2}}{k}} \\
X_{j}[i] & =\frac{x_{j}[i]-m_{i}}{s_{i}} \quad \text { for } \quad j=1, \ldots, k, i=1, \ldots, n
\end{aligned}
$$

where $n$ defines the number of features per pattern, $m_{i}$ and $s_{i}$ are the mean and the variance of the $i$ th feature, $x_{j}$ is the unprocessed $j$ th pattern, $k$ defines the number of patterns contained in the model database, and $X_{j}$ represents the normalized $j$ th pattern. The classification stage computes the Euclidean distance between the input candidate surface and the surfaces contained in the polyp and fold databases

$$
\operatorname{dist}_{j}=\sqrt{\sum_{i=1}^{n}\left(X_{j}[i]-Y[i]\right)^{2}} \text { for } i=1, \ldots, n
$$

where $X_{j}$ is the $j$ th pattern from the database and $Y$ defines the normalized feature pattern derived from the candidate surface. The candidate surface is classed as polyp if the $\min _{j}\left(d i s t_{j}\right)$ is given by a surface from the polyp database, otherwise is classed as fold.

Our training data consist of polyp and fold databases that were generated and validated by three radiologists from the Mater Misericordiae Hospital, Dublin. To avoid classification bias the datasets used in the generation of the polyp and fold databases (six patient datasets and three synthetic datasets) were not included in the experimental evaluation presented in the next section. While only a small number of datasets used in our evaluation contained polyps, the classification results obtained using the leave-one-patient out approach would be biased toward datasets with a large number of polyps. To avoid this problem, in this study we have employed a comprehensive classification procedure where a fixed number of polyps and folds that were deemed representative by our clinical partners were used to train the system. During the training stage we segregated the polyps into small spherical, medium spherical, large spherical, and elliptical subclasses. We also segregated the folds into small folds, small convex surfaces, medium folds, and large folds. We have chosen to divide these databases by size in order to alleviate as much as possible the class overlapping in the training stage. For training we used 58 polyps $(36.21 \%<5 \mathrm{~mm}, 22.41 \%$ in the range $[5-10 \mathrm{~mm}]$ and $41.38 \%$ $>10 \mathrm{~mm})$ and 247 folds $(29.15 \%<5 \mathrm{~mm}, 34.01 \%$ in the range [5-10 mm], $21.86 \%>10 \mathrm{~mm}$ and $14.98 \%$ surfaces with low convexity). This class segregation by size offered the optimal solution to increase the correct identification rate for small and medium polyps, but this advantage has not been obtained at the expense of increasing the level of false positives. Experimental data indicate that our FNNN classifier provides superior performance to that of the more established PNN classifier.

\section{EXPERIMENTS AND RESULTS}

\section{A. Evaluation of the Automated Colon Segmentation}

We have applied the colon segmentation algorithm detailed in Section III on 152 (supine and prone) datasets acquired using a Siemens Somatom Sensation 4/16 slice CT scanner with standard radiation dose (100 mAs) and 27 low-dose datasets (radiation dose in the range 13-40 mAs). In 63 standard dose and 14 low-dose datasets the colon was fully distended and was detected by the segmentation algorithm without inclusion of any extra colon surface (ECS). Consequently, the colon surface detection was $100 \%$, and the ECS error was $0 \%$. As detailed in Section III the detection of the collapsed colons was performed in several phases. The segmentation algorithm starts with the identification of large segments (with V/L > 300) and during this stage 223 disjoint air regions were detected in 84 standard-dose datasets and 31 large segments were detected in 13 low-dose datasets. Out of these 254 regions, 252 were colon parts and two regions were sections of the small intestine. The detection of small regions $(\mathrm{V} / \mathrm{L}<300)$ was performed in the second phase. In total 352 small air regions with $\mathrm{V} / \mathrm{L}<300$ were detected in 84 datasets, of which, 161 were colon surfaces and 191 were ECSs and 12 small colon segments were undetected. In 13 low-dose datasets, 48 small air regions were detected, of which 25 were colon segments, 23 were ECSs, and 4 colon segments were undetected. In 84 collapsed colons, the colon surface detection was higher than $95 \%$. Only in 5 cases it was less than $98 \%$ (95.51 and $97.71 \%$, respectively), and in three cases it was between 98-99\%. The largest ECS inclusion was $14.24 \%$ with a mean of $1.61 \%$. For 13 low-dose datasets with collapsed colon CT data, 9 had no ECS inclusion and the colon reconstruction was complete (100\%), 2 had less than $0.20 \%$ ECS inclusion, one had $6.5 \%$ ECS inclusion, 1 had $4.7 \%$ ECS inclusion and $96.3 \%$ surface detection. For one dataset the algorithm failed to identify approximately $13 \%$ of the colon surface with no ECS inclusion.

To validate our automatic colon segmentation algorithm, radiologists from Mater Hospital performed a manual segmentation using a multiseed region growing algorithm; this provided the ground truth data. The manual segmentation was performed in conjunction with a 3-D visualization software tool that allowed radiologists to use all available information in deciding whether 
TABLE III

PERFORMANCE OF THE CAD-CTC SySTEM WHEN APPLIED TO REAL PATIENT DATA

\begin{tabular}{|c|c|c|c|c|c|}
\hline \multirow{2}{*}{ Type } & \multirow{2}{*}{ No } & \multicolumn{2}{|c|}{ FNNN } & \multicolumn{2}{c|}{ PNN } \\
\cline { 3 - 6 } & & TP & Sen. & TP & Sen. \\
\hline$>=10 \mathrm{~mm}$ & 4 & 4 & $100 \%$ & 4 & $100 \%$ \\
\hline$[5-10) \mathrm{mm}$ & 25 & 23 & $92 \%$ & 21 & $84 \%$ \\
\hline$<5 \mathrm{~mm}$ & 84 & 48 & $57.14 \%$ & 43 & $51.19 \%$ \\
\hline Mass & 11 & 8 & $72.72 \%$ & 4 & $36.36 \%$ \\
\hline Flat & 3 & 2 & $66.67 \%$ & 0 & $0 \%$ \\
\hline Total & 127 & 85 & $66.93 \%$ & 72 & $56.69 \%$ \\
\hline FP & & & 3.38 & & 2.15 \\
\hline
\end{tabular}

an air-filled region is part of the colon or not. We compared the results returned by our automated colon segmentation with this ground truth data.

To prove the validity of our approach we compared its performance with the performance returned by other colon segmentation techniques. In this regard, the method proposed by Nappi et al. [17] was able to identify the collapsed colons with an average of $12.5 \%$ ECS inclusion and with $99.1 \%$ detection of the colon surface. The colon segmentation technique detailed by Iordanescu et al. [11] achieved only $83.2 \%$ success rate for complete colon segmentation. Our technique achieved 97.20\% success rate for complete colon segmentation where the average ECS inclusion is $1.61 \%$ with $99.68 \%$ detection of the colon surface when applied to 152 datasets (the algorithm produced inappropriate results when applied to five datasets that were generated by patients that were subjected to an improper bowel preparation-more than $50 \%$ of the colon area was filled with fluid and residual material). Frimmel et al. [34] method uses the centerline and the colon geometry for automatic segmentation and shows $96 \%$ sensitivity for automatic colon segmentation.

\section{B. Evaluation of the Polyp Detection Algorithm}

In our tests we have used 53 patient datasets (87 prone and supine views) with 127 polyps, 21 low-dose patient datasets (26 supine and prone views) with 17 polyps, five patient datasets with 33 synthetic polyps [24], and a phantom dataset with 48 polyps of various sizes. Overall sensitivities (Sen.) for true polyp (TP) detection were $66.93 \%$ and $56.69 \%$ when the FNNN and PNN classifiers were employed (see Table III). The false positive rates were 3.38 and 2.15 for FNNN and PNN classifiers respectively. The sensitivity of the system for the detection of the clinical significant polyps in the range 5-10 $\mathrm{mm}$ was $92 \%$ (FNNN) and $84 \%(\mathrm{PNN})$.

When the CAD-CTC system was applied to real patient datasets with synthetic generated polyps, the overall sensitivities for FNNN and PNN classifiers was $84.85 \%$ with false positives per dataset of 2.6 and 1.8, respectively. The overall sensitivities of our CAD-CTC system when applied to phantom data were $89.36 \%$ (FNNN) and $85.41 \%$ (PNN) (see Table V).

To determine whether a polyp was correctly detected by our CAD-CTC system, we cross-validated the location of the detected polyps with the CTC reports prepared by the radiologists from the Mater Hospital (the CTC reports were used as the primary ground truth data). We also compared the results returned by the CAD-CTC system with the colonoscopy reports in cases when we noted disagreements between the CTC reports (i.e., polyps that have not been identified in all CTC reports).
TABLE IV

PERFORMANCE OF THE CAD-CTC SYSTEM WHEN APPLIED TO SYNTHETIC DATA

\begin{tabular}{|l|c|c|c|c|c|}
\hline \multirow{2}{*}{ Type } & \multirow{2}{*}{ No } & \multicolumn{2}{|c|}{ FNNN } & \multicolumn{2}{c|}{ PNN } \\
\cline { 3 - 6 } & & TP & Sen. & TP & Sen. \\
\hline$>10 \mathrm{~mm}$ & 9 & 9 & $100 \%$ & 9 & $100 \%$ \\
\hline$[5-<10) \mathrm{mm}$ & 17 & 17 & $100 \%$ & 17 & $100 \%$ \\
\hline$<5 \mathrm{~mm}$ & 6 & 2 & $33.33 \%$ & 2 & $33.33 \%$ \\
\hline Flat & 1 & 0 & $0 \%$ & 0 & $0 \%$ \\
\hline Total & 33 & 28 & $84.85 \%$ & 28 & $84.85 \%$ \\
\hline FP & & & 2.6 & & 1.8 \\
\hline
\end{tabular}

TABLE V

PERFormanCE OF THE CAD-CTC SYSTEM WHEN APPLIED TO STANDARD DOSE PHANTOM DATA (100 MAS)

\begin{tabular}{|l|c|c|c|c|c|}
\hline \multirow{2}{*}{ Type } & \multirow{2}{*}{ No } & \multicolumn{2}{|c|}{ FNNN } & \multicolumn{2}{c|}{ PNN } \\
\cline { 3 - 6 } & & TP & Sen. & TP & Sen. \\
\hline$>=10 \mathrm{~mm}$ & 14 & 14 & $100 \%$ & 13 & $92.85 \%$ \\
\hline$[5-10) \mathrm{mm}$ & 20 & 20 & $100 \%$ & 20 & $100 \%$ \\
\hline$<5 \mathrm{~mm}$ & 5 & 5 & $100 \%$ & 5 & $100 \%$ \\
\hline Flat & 9 & 4 & $44.44 \%$ & 3 & $33.33 \%$ \\
\hline Total & 48 & 43 & $89.36 \%$ & 41 & $85.41 \%$ \\
\hline FP & & & 1 & & 1 \\
\hline
\end{tabular}

One of the main concerns in adopting CTC as a screening technique for colorectal polyp detection is the patient exposure to ionising radiation [28]. Since a reduction in radiation dose will increase the level of noise in the CT data, our aim is to fully evaluate the impact of the radiation dose on the performance of our CAD-CTC system. To address this issue, an artificial phantom developed by our group [27] was scanned with different radiation doses. The phantom used in our experiments has 48 latex polyps of varying shapes (pedunculated, sessile, and flat) with sizes in the range 3 to $18 \mathrm{~mm}$. The synthetic phantom has been scanned with radiation doses of $100 \mathrm{mAs}$ (see Table IV), 40, 20, and $13 \mathrm{mAs}$ (see Table V). The experimental results are summarized in Table VI.

It can be observed that the polyp detection performance of our CAD-CTC system is virtually unchanged at radiation doses as low as $13 \mathrm{mAs}$ (we would expect the algorithm to produce high sensitivity values when applied to phantom data due to the artificial nature of the latex polyps). To fully evaluate the impact of the radiation dose we also applied our polyp detection CAD system to 26 real patient low-dose data (11 datasets scanned at $13 \mathrm{mAs}$, seven datasets scanned at $20 \mathrm{mAs}$, seven datasets scanned at $30 \mathrm{mAs}$, and one dataset scanned at $40 \mathrm{mAs}$ ). The associated polyp detection performance is illustrated in Table VII.

\section{COMPARISON With OTHER CAD-CTC SYSTEMS}

The CAD-CTC system developed by Summers et al. [26] attempts to identify the polyps in the CT data using a multistage geometrically driven approach. Initially, they detect the convex surfaces that protrude inward from the colon by applying a kernel filter that is constructed using partial derivatives. After the detection of candidate surfaces, they use shape-based criteria including the principle curvature $\left(k_{\min }\right.$ and $\left.k_{\max }\right)$, mean curvature $(\mathrm{H})$, the sphericity ratio of the candidate surface $s=$ $\left(k_{\max }-k_{\min }\right) / H$, and the minimum polyp size. In order to reduce the level of false positives they employed a very restrictive shape criteria, but the reported sensitivity for all sizes of polyps 
TABLE VI

PERFORMANCE OF THE CAD-CTC WHEN APPLIED TO LOW-DOSE PHANTOM DATASETS

\begin{tabular}{|l|c|c|c|c|}
\hline Size & mAs & Total & TP & $\begin{array}{c}\text { Sensitivity } \\
\text { (FNNN) }\end{array}$ \\
\hline$>10 \mathrm{~mm}$ & 13 & 14 & 14 & $100 \%$ \\
\hline$[5-10) \mathrm{mm}$ & 13 & 20 & 20 & $100 \%$ \\
\hline$<5 \mathrm{~mm}$ & 13 & 5 & 5 & $100 \%$ \\
\hline Flat & 13 & 9 & 4 & $44.4 \%$ \\
\hline Total & & 48 & 43 & $89.95 \%$ \\
\hline FP & & & & 3 \\
\hline
\end{tabular}

\begin{tabular}{|l|c|c|c|c|}
\hline Size & mAs & Total & TP & $\begin{array}{c}\text { Sensitivity } \\
\text { (FNNN) }\end{array}$ \\
\hline$>10 \mathrm{~mm}$ & 20 & 14 & 14 & $100 \%$ \\
\hline$[5-10) \mathrm{mm}$ & 20 & 20 & 19 & $100 \%$ \\
\hline$<5 \mathrm{~mm}$ & 20 & 5 & 5 & $95 \%$ \\
\hline Flat & 20 & 9 & 4 & $44.44 \%$ \\
\hline Total & & 48 & 39 & $87.5 \%$ \\
\hline FP & & & & 2 \\
\hline
\end{tabular}

\begin{tabular}{|l|c|c|c|c|}
\hline Size & mAs & Total & TP & $\begin{array}{c}\text { Sensitivity } \\
\text { (FNNN) }\end{array}$ \\
\hline$>10 \mathrm{~mm}$ & 40 & 14 & 14 & $100 \%$ \\
\hline$[5-10) \mathrm{mm}$ & 40 & 20 & 20 & $100 \%$ \\
\hline$<5 \mathrm{~mm}$ & 40 & 5 & 5 & $100 \%$ \\
\hline Flat & 40 & 9 & 4 & $44.44 \%$ \\
\hline Total & & 48 & 43 & $89.95 \%$ \\
\hline FP & & & & 1 \\
\hline
\end{tabular}

TABLE VII

Performance of THE CAD-CTC WhEN APPLIED TO LOW-DOSE PATIENT CT DATA

\begin{tabular}{|l|c|c|c|}
\hline \multirow{2}{*}{ Type } & \multirow{2}{*}{ No } & \multicolumn{2}{|c|}{ FNNN } \\
\cline { 3 - 4 } & & TP & Sensitivity \\
\hline$>=10 \mathrm{~mm}$ & 5 & 5 & $100 \%$ \\
\hline$[5-10) \mathrm{mm}$ & 9 & 8 & $88.89 \%$ \\
\hline$<5 \mathrm{~mm}$ & 3 & 2 & $66.67 \%$ \\
\hline Total & 17 & 15 & $88.24 \%$ \\
\hline FP & & & 3.65 \\
\hline
\end{tabular}

was influenced by the selection of the kernel filter. The performance achieved when the optimal filter was employed showed $71 \%$ sensitivity for large polyps with 3.4 FPs per dataset.

The polyp detection system developed by Nappi and Yoshida [18] employs the shape index (SI) and the curvature (CV) to identify the candidate convex surfaces. In this regard, the shape index is divided into five different categories, and the magnitude of the effective curvature is used to sample the surface convexity for the voxel under investigation. They compared the SI and $\mathrm{CV}$ values with experimentally detected thresholds. From candidate surfaces they evaluate the Gaussian concentration (GC) that measure the concentration of the gradient vectors around the voxel of interest using the assumption that for spherical surfaces the GC is highest at the center of the sphere. Their CAD system shows $100 \%$ sensitivity for polyps larger than $10 \mathrm{~mm}$ with 1.7 FP per dataset.

Paik et al. [20] developed an algorithm called surface normal overlap that was applied to colorectal polyp detection. Their algorithm is based on the assumption that the colorectal polyps are convex structures and the local normal intersection density samples the local convexity for each voxel of the colon wall. The normal overlap technique was used to identify suspicious convex structures while the polyp detection is performed by assessing the deviation of these convex structures from a stochastic model employed to define the shape of a nominal polyp. This algorithm shows $100 \%$ sensitivity in detecting polyps larger than $10 \mathrm{~mm}$ with $7 \mathrm{FP}$ datasets. No experimental data is provided in regard to the sensitivity of their CAD-CTC system when applied to the identification of small $(<5 \mathrm{~mm})$ and mid-sized polyps (between 5-10 mm).

Acar et al. [1] employed a different approach based on the edge flow displacement that is applied to obtain robust polyp detection. They developed a method to extract the candidate surfaces based on the Hough transform that evaluates the normal intersections using the assumption that the normal intersection will be high for convex (cap-like) structures. After the extraction of the candidate surfaces, they scrolled these surfaces with a plane perpendicular on the main axis of the surface and they computed the edge flow from the extremity of the surface toward its center. The divergence of the edge flow is used to class the candidate surface into a polyp or a fold generated surface. They applied this technique on 48 datasets and their experiments indicated that their method achieved 35\% specificity at a sensitivity rate of $100 \%$. This method was further advanced by Gokturk et al. [9] when they applied the randomly oriented triple orthogonal planes at the location of each candidate surface. They applied this approach to sample the sphericity of the candidate surface based on the fact that any random plane slicing trough a spherical surface will generate a circle. The experimental results indicate that their technique achieved $69 \%$ specificity at a sensitivity rate of $100 \%$. No detailed analysis with respect to the size of the polyps is provided.

Kiss et al. [15] analyzed the slope density function as a discriminative feature to classify the convex candidate surfaces into polyps and folds. The initial stage of their system identifies the candidate surfaces by intersecting the colon wall with a reformatted plane perpendicular on the local normal surface. If the intersection patch between the reformatted plane and the colon surface is filled with voxel data the colon surface is concave and is declared part of the healthy colon tissue. Otherwise it is a convex surface that is generated either by polyps or folds. The resulting candidate surfaces are evaluated statistically using the slope density function, which shows peaks for elongated surfaces and smooth values for ellipsoidal surfaces. This property of the SDF is very useful as it provides robust discrimination between the polyps and folds. Their method obtained the following performance in polyp detection: $33.33 \%$ sensitivity for polyps smaller than $5 \mathrm{~mm}, 85.70 \%$ for polyps in the range 6-9 $\mathrm{mm}$ and $90 \%$ for polyps larger than $9 \mathrm{~mm}$.

It can be observed that the performance of the CAD-CTC system detailed in this paper compares well with those analyzed above (see Table VIII for a summary of the performance of the most relevant documented CAD-CTC systems). The results reported in the first three rows of Table VIII are based on manual (radiologist) evaluation of CTC data, the remaining cases relate to automated CAD-CTC systems. The performance in polyp detection achieved by the automated CAD-CTC systems closely match or even outperform the performance in polyp detection achieved by manual CTC techniques but the main problem associated with most CAD-CTC systems is the high level of false positives. However, it is important to mention that the results illustrated in Table VIII are only indicative as it is difficult to fully 
TABLE VIII

Summary Performance of the SeleCted CTC AND CAD-CTC Systems

\begin{tabular}{|c|c|c|c|c|c|c|c|c|c|}
\hline Technique / Authors & \# Patients & Positioning & $\begin{array}{l}\text { \# Data } \\
\text { Sets }\end{array}$ & \# Polyps & $\begin{array}{l}\text { Dose } \\
\text { (mAs) }\end{array}$ & $\begin{array}{l}\text { Polyp Size } \\
\text { (mm) }\end{array}$ & Sensitivity (\%) & $\begin{array}{l}\text { False Positives } \\
\text { Per Patient }\end{array}$ & $\begin{array}{l}\text { Specificity } \\
(\%)\end{array}$ \\
\hline $\begin{array}{l}\text { CTC } \\
\text { (Fenlon, 1999) }\end{array}$ & 87 & & & $\begin{array}{l}22 \\
40 \\
53 \\
115 \\
\end{array}$ & & $\begin{aligned} 210 \\
6-9 \\
1-5 \\
\text { All Sizes }\end{aligned}$ & $\begin{array}{l}91 \\
82 \\
55 \\
71 \\
\end{array}$ & $\begin{array}{c}2 \\
8 \\
9 \\
19 \\
\end{array}$ & \\
\hline $\begin{array}{l}\text { CTC (3D) } \\
\text { (Pickhardt, 2003) }\end{array}$ & 1233 & P\&S & & & & $\begin{array}{l}\geq 10 \\
\geq 8 \\
\geq 6\end{array}$ & $\begin{array}{l}93.8 \\
93.9 \\
88.7 \\
\end{array}$ & & $\begin{array}{c}96 \\
92.2 \\
79.6 \\
\end{array}$ \\
\hline $\begin{array}{l}\text { CTC Low Dose } \\
\text { (Van Gelder et al., 2002) }\end{array}$ & & & & $\begin{array}{l}49 \\
13\end{array}$ & $\begin{array}{l}100 \\
30\end{array}$ & $\begin{array}{l}<5 \\
\geq 5 \\
<5 \\
\geq 5 \\
\end{array}$ & $\begin{array}{l}24 \\
85 \\
24 \\
85 \\
\end{array}$ & & \\
\hline $\begin{array}{l}\text { Random Orthogonal Shape } \\
\text { Selection } \\
\text { (Gokturk et al., 2001) }\end{array}$ & 48 & P\&S & 48 & & & & $\begin{array}{l}100 \\
95\end{array}$ & & $\begin{array}{l}69 \\
74\end{array}$ \\
\hline $\begin{array}{l}\text { Edge Displacement Fields } \\
\text { (Acar et al., 2002) }\end{array}$ & 48 & P\&S & 48 & & & $2-15$ & $\begin{array}{l}100 \\
95 \\
90 \\
80 \\
\end{array}$ & & $\begin{array}{l}40 \\
20 \\
\sim 0 \\
\sim 0\end{array}$ \\
\hline $\begin{array}{l}\text { Surface Normal Overlap } \\
\text { Paik (2004) }\end{array}$ & 8 & so & 8 & & & $\geq 10$ & $\begin{array}{l}100 \\
90 \\
80\end{array}$ & $\begin{array}{l}7.0 \\
6.0 \\
4.6\end{array}$ & \\
\hline $\begin{array}{l}\text { Normal } \\
\text { (Kiss et al., 2002) }\end{array}$ & 18 & $\mathrm{NA}$ & 18 & & & $\begin{array}{c}5-9 \\
\geq 10 \\
\text { All Sizes }\end{array}$ & $\begin{array}{l}20 \\
100 \\
60\end{array}$ & $\begin{array}{l}8.17 \\
3.76 \\
3.17\end{array}$ & \\
\hline $\begin{array}{l}\text { Normal / Slope Density Function } \\
\text { (Kiss et al., 2003) }\end{array}$ & & & 50 & \begin{tabular}{|c|}
6 \\
8 \\
10 \\
12 \\
4 \\
40 \\
\end{tabular} & & $\begin{array}{c}\text { Flat } \\
<5 \\
6-9 \\
>9 \\
\text { Tumor } \\
\text { All Sizes } \\
\end{array}$ & $\begin{array}{c}20 \\
33 \\
85.7 \\
90.90 \\
100 \\
69.69 \\
\end{array}$ & $2.48 /$ dataset & \\
\hline $\begin{array}{l}\text { Surface Curvature } \\
\text { (Summers et al., 2001) }\end{array}$ & & P\&S & & $\begin{array}{l}28 \\
12 \\
10 \\
50\end{array}$ & & $\begin{array}{c}\geq 10 \\
5-9 \\
<5 \\
\text { All Sizes }\end{array}$ & 71 & $3.4 /$ dataset & \\
\hline $\begin{array}{l}\text { Surface Curvature } \\
\text { (Jerebko et al., 2003) }\end{array}$ & 40 & P\&S & 80 & & & $>3$ & 100 & 3 & \\
\hline $\begin{array}{l}\text { Fuzzy clustering and deformable } \\
\text { models } \\
\text { (Yao et al., 2004) }\end{array}$ & 20 & P\&S & 40 & & & $3-30$ & $\begin{array}{c}100 \\
80\end{array}$ & $\begin{array}{l}11.5 \\
6.5\end{array}$ & \\
\hline $\begin{array}{l}\text { 3D geometric Features } \\
\text { (Nappi \& Yoshida, 2002) }\end{array}$ & 43 & P\&S & & 12 & & $5-30$ & $\begin{array}{c}100 \\
95\end{array}$ & $\begin{array}{l}2.4 / \text { dataset } \\
1.7 / \text { dataset }\end{array}$ & \\
\hline $\begin{array}{l}\text { 3D geometric Features } \\
\text { (Nappi \& Yoshida, 2003) }\end{array}$ & 72 & & & 21 & & $\geq 10$ & $\begin{array}{c}100 \\
95\end{array}$ & $\begin{array}{l}1.5 / \text { dataset } \\
1.3 / \text { dataset }\end{array}$ & \\
\hline
\end{tabular}

evaluate them since the proposed systems have been applied to different datasets acquired with differing preparation procedures and scanning protocols that impact on the resolution of the CT data. The CT data used in our experiments has been acquired with a relatively low reconstruction interval $(1.5 \mathrm{~mm})$, and we believe that the performance of our system will improve when it will be applied to higher resolution CT data since the partial volume effects are less pronounced. [the CT data used in our experiments (see Section VI) have been acquired using a Siemens Somatom Sensation multislice CT scanner where the following protocol was employed: collimation $2.5 \times 4 \mathrm{~mm}$ (4 slices) and $1.5 \times 16 \mathrm{~mm}$ (16 slices), table speed $30 \mathrm{~mm} /$ rotation, reconstruction interval $1.5 \mathrm{~mm}$, slice thickness $3 \mathrm{~mm}$, tube voltage $120 \mathrm{kVp}$, effective tube current $100 \mathrm{mAs}$ (standard dose), and 13-40 mAs(low-dose)]. The lack of publicly available standard test datasets makes the comparison of these CAD-CTC systems difficult. In order to address this issue we have applied our CAD-CTC system to the Walter-Reed datasets [33], and the performance in polyp detection of our system is depicted in Table IX. (The Walter-Reed CT data have been acquired using a four- and eight-channel General Electric (GE) Light Speed scanner where the following protocol was employed: collimation 1.25 to $2.5 \mathrm{~mm}$, table speed $15 \mathrm{~mm} /$ rotation, reconstruction interval $1 \mathrm{~mm}$, slice thickness $1.25 \mathrm{~mm}$, tube voltage $120 \mathrm{kVp}$, effective tube current $100 \mathrm{mAs}$.)
TABLE IX

Performance of OUR CAD-CTC SySTEM When APPLIED TO THE WALTER-REED DATABASE

\begin{tabular}{|l|c|c|c|}
\hline Size & Total & TP & Sensitivity (FNNN) \\
\hline$>10 \mathrm{~mm}$ & 15 & 15 & $100 \%$ \\
\hline$[5-10) \mathrm{mm}$ & 31 & 28 & $90.32 \%$ \\
\hline$<5 \mathrm{~mm}$ & 5 & 3 & $60.0 \%$ \\
\hline Flat & 3 & 1 & $33.33 \%$ \\
\hline Total & 54 & 47 & $87.03 \%$ \\
\hline FP & & & 2.17 \\
\hline
\end{tabular}

The CAD-CTC system detailed in this paper is able to produce consistent results when applied to a large number of datasets that are acquired using different CT scanners and different image acquisition protocols. The adoption of standard databases will allow a more detailed comparison of polyp detection performance achieved by competing CAD-CTC techniques.

\section{CONCLUSION}

The aim of this paper is the description of a fully integrated CAD polyp detection system. The main problem associated with fully automated CAD-CTC systems is the large amount of false positives that are reported by the system, a fact that makes the application of these systems to clinical studies impractical. The 
TABLE X

Statistics of THE SuRfaces That Generated FALSE Positives

\begin{tabular}{|c|c|c|}
\hline $\begin{array}{c}\text { Cause that generated } \\
\text { false positives }\end{array}$ & $\begin{array}{c}\text { Number of false } \\
\text { positives }\end{array}$ & $\%$ \\
\hline Folds & 145 & 45.74 \\
\hline Small convex surfaces & 112 & 35.33 \\
\hline Residual material & 37 & 11.67 \\
\hline Inflation tube & 23 & 7.25 \\
\hline
\end{tabular}

system detailed in this paper is able to identify the polyps with an overall sensitivity of $66.93 \%$ with $3.38 \mathrm{FP}$ per dataset and the identification of clinically relevant polyps $>5 \mathrm{~mm}$ is obtained with a sensitivity of $93.10 \%$. The overall sensitivity attained by our system is lowered by the relative low sensitivity achieved in detecting the polyps smaller than $5 \mathrm{~mm}(57.14 \%)$. This low sensitivity rate is justified by the fact that the surfaces generated by small polyps are defined by a very small number of voxels and an increase in the detection of small polyps will be achieved at the expense of increasing significantly the number of false positives. In fact the detection of polyps smaller than $5 \mathrm{~mm}$ is problematic even in cases when the CT data is manually evaluated by radiologists. This can be observed in Table VIII where it is indicated that the sensitivity in detecting the polyps smaller than $5 \mathrm{~mm}$ is only $55 \%$ with nine false positives per dataset [7] (see the first row in Table VIII). This indicates that the performance of our system in detecting small polyps $(<5 \mathrm{~mm})$ is comparable to the performance of manual CTC. The standard patient preparation procedure utilized by our clinical colleagues (Mater Hospital) does not include the administration of contrast agent for faecal tagging. The lack of faecal tagged data precluded the application of digital subtraction algorithms to remove the small residual material that is attached to the colon, and this fact increased artificially the level of false positives especially for polyps smaller than $5 \mathrm{~mm}$. We believe that a reduction in false positives is still possible if the patient preparation includes the administration of contrast agent for faecal tagging. A statistical categorization of the false positives with respect to the cause that produced them is illustrated in Table X.

Most of the false positives are generated by the large fold structures with complex morphologies $(45.74 \%)$ that are incorrectly divided in multiple surfaces by the candidate surface extraction technique. The false positives generated by the fold surfaces can be categorized by size as follows: 67 were generated by folds larger than $10 \mathrm{~mm}(46.21 \%), 53$ by medium sized folds [5-10 $\mathrm{mm}](36.55 \%)$ and 25 by folds smaller than $5 \mathrm{~mm}(17.24 \%)$. Another source of false positives are the small convex surfaces (smaller than $5 \mathrm{~mm}$ ) that are generated by the partial volume effects (these are caused by the low resolution in the $z$-axis). In our experiments all datasets supplied by our clinical partners are scanned with $1.5-\mathrm{mm}$ reconstruction interval, and higher resolution data will lead to a reduction in false positives. Another important finding resulting from this investigation is the fact that the radiation dose does not have a severe impact on the performance in polyp detection of our CAD-CTC system. A large number of experiments have been conducted using datasets obtained by scanning a synthetic phantom with different radiation doses and low-dose patient data, and we conclude that robust polyp detection is possible even at radiation doses as low as $13 \mathrm{mAs} /$ rotation. The time required by our system to process completely one dataset is in the range 7 to $8 \mathrm{~min}$, and this time is significantly lower than the time required to analyze the data manually.

Our system is fully integrated, and we believe that its performance makes it suitable to be applied in clinical examinations. Our prototype CAD-CTC is currently in deployment at the Mater Misericordiae Hospital (Ireland).

\section{ACKNOWLEDGMENT}

The authors would like to acknowledge the contribution of our clinical partners Dr. Helen Fenlon, Dr. Alan O’Hare (Department of Radiology), and Dr. Padraic MacMathuna (Gastrointestinal Unit) from Mater Misericordiae Hospital, Dublin, Ireland. The authors would also like to acknowledge the valuable input from our colleagues from the Vision Systems Group, namely, Robert Sadleir and Nicolas Sezille.

\section{REFERENCES}

[1] B. Acar, C. F. Baeulieu, S. B. Gokturk, C. Tomasi, D. S. Paik, R. B. Jeffrey, J. Yee, and S. Napel, "Edge displacement field-based classification for improved detection of polyps in CT colonography," IEEE Trans. Med. Imag., vol. 21, no. 12, pp. 1461-1467, Dec. 2001.

[2] American Cancer Society, Cancer Facts and Figures 1999.

[3] Cancer Research UK, Bowel Cancer Factsheet April 2003.

[4] F. R. David and S. Robert, "Screening for colorectal cancer," N. Engl. J. Med., vol. 346, no. 1, Jan. 2002.

[5] E. W. Dijkstra, "A note on two problems in connection with graphs," Numer. Math., vol. 1, pp. 269-271, 1959.

[6] H. M. Fenlon and J. T. Ferucci, "Virtual colonoscopy: What will this issue be?," Amer. J. Roent., vol. 169, no. 2, pp. 453-458, 1997.

[7] H. M. Fenlon, D. P. Numes, P. C. Schroy, M. A. Barish, P. D. Clarke, and J. T. Ferrucci, "A comparison of virtual and conventional colonoscopy for the detection of colorectal polyps," N. Engl. J. Med., vol. 341, no. 20, pp. 1496-1503, 1999.

[8] O. Ghita and P. F. Whelan, "A bin picking system based on depth from defocus," Mach. Vis. Appl., vol. 13, no. 4, pp. 234-244, 2003.

[9] B. Gokturk, C. Tomasi, B. Acar, C. Beaulieu, D. Paik, R. B. Jeffrey, J. Yee, and S. Napel, "A statistical 3D pattern processing method for computer aided detection of polyps in CT colonography," IEEE Trans. Med. Imag., vol. 20, no. 12, pp. 1251-1260, Dec. 2001.

[10] A. K. Hara, C. D. Johnson, J. E. Reed, D. A. Ahlquist, H. Nelson, R. L. MacCarty, W. S. Harmsen, and D. M. Ilstrup, "Detection of colorectal polyps with CT colography: Initial assessment of sensitivity and specificity," Radiology, vol. 205, no. 1, pp. 59-65, 1997.

[11] G. Iordanescu, P. J. Pickhardt, J. R. Choi, and R. M. Summers, "Automated seed placement for colon segmentation in CT colonography," Acad. Rad., vol. 12, pp. 182-190, 2005.

[12] A. K. Jerebko, J. D. Malley, M. Franaszek, and R. M. Summers, "Support vector machines committee classification method for computeraided polyp detection in CT colonography," Acad. Rad., vol. 12, pp. 479-486, 2005.

[13] C. D. Johnson and A. K. Hara, "CT colonography: The next colon screening examination?,” Radiology, vol. 216, pp. 331-341, 2000.

[14] G. Kiss, J. Van Cleynenbreugel, M. Thomeer, P. Suetens, and G. Marchalc, "Computer-aided diagnosis in virtual colonography via combination of surface normal and sphere fitting methods," Eur. Rad., vol. 12, no. 1, pp. 77-81, 2002.

[15] G. Kiss, J. Van Cleynenbreugel, P. Suetens, and G. Marchal, "Computer aided diagnosis for CT colonography via slope density functions," in MICCAI 2003, Montréal, QC, Canada, Nov. 2003.

[16] Least Squares Approximation-Web Documentation [Online]. Available: http://www.magic-software.com/Documentation.html

[17] J. Nappi, A. H. Dachman, P. MacEneaney, and H. Yoshida, "Automated knowledge-guided segmentation for colonic walls for computerized detection in CT colonography," J. Comput. Assist. Tomogr., vol. 26, no. 4, pp. 493-504, 2002.

[18] J. Nappi and H. Yoshida, "Automated detection of polyps with CT colonography: Evaluation of volumetric features for reduction of false positive findings," Acad. Rad., vol. 9, no. 4, pp. 386-397, 2002. 
[19] NCRI, Cancer in Ireland, Incidence and Mortality Healy \& Associates, 2002.

[20] D. S. Paik, C. F. Beauilieu, G. D. Rubin, B. Acar, R. B. Jefferey, J. Yee, J. Dey, and S. Napel, "Surface normal overlap: A computer aided detection algorithm with application to colonic polyps and lung nodules in helical CT," IEEE Trans. Med. Imag., vol. 23, no. 6, pp. 661-675, 2004.

[21] S. Parker, T. Tong, S. Bolden, and P. Wingo, "Cancer statistics 1997," CA Cancer J. Clin., vol. 47, pp. 5-27, 1997.

[22] P. J. Pickhardt, J. R. Choi, I. Hwang, J. A. Butler, M. L. Puckett, and H. A. Hildebrandt, "Computed tomographic virtual colonoscopy to screen for colorectal neoplasia in asymptomatic adults," N. Engl. J. Med., vol. 349, no. 23, pp. 2191-2200, 2003.

[23] R. J. T. Sadleir and P. F. Whelan, "Fast colon centreline calculation using optimised 3D topological thinning," Comput. Med. Imaging Graph., vol. 29, no. 4, pp. 251-258, 2005.

[24] N. Sezille, R. J. T. Sadleir, and P. F. Whelan, "Automated synthesis, insertion and detection of polyps for CT colonography," in OPTO-Ireland SPIE's Regional Meeting on Optoelectronics, Photonics and Imaging, Galway, Ireland, 2002, pp. 183-191.

[25] R. M. Summers, C. F. Beaulieu, L. M. Pusanik, J. D. Malley, R. B. Jeffrey, D. I. Glazer, and S. Napel, "Automated polyp detector for CT colonography feasibility study," Radiology, vol. 216, pp. 284-290, 2000.

[26] R. M. Summers, C. D. Johnson, L. M. Pusanik, J. D. Malley, A. M. Youssef, and J. E. Reed, "Automated polyp detection at CT colonography: Feasibility assessment in a human population," Radiology, vol. 219, pp. 51-59, 2001.

[27] T. A. Chowdhury, P. F. Whelan, O. Ghita, N. Sezille, and S. Foley, "Development of a synthetic phantom for the selection of optimal scanning parameters in CAD-CT colonography," Med. Eng. Phys. Nov. 9, 2006.

[28] R. E. van Gelder, H. W. Venema, I. W. Serlie, C. Y. Nio, R. M. Determann, C. A. Tipker, F. M. Vos, A. S. Glas, J. F. W. Bartelsman, P. M. Bossuyt, J. S. Lameris, and J. Stoker, "CT colonography at different radiation dose levels: Feasibility of dose reduction," Radiology, vol. 224, pp. 25-33, 2002.

[29] D. J. Vining, "Virtual colonoscopy," Gastrointest. Endosc. Clin. North Amer., vol. 7, pp. 285-291, 1997.

[30] J. Yao, M. Miller, M. Franaszek, and R. M. Summers, "Colonic polyp segmentation in CT colonography based on fuzzy clustering and deformable models," IEEE Trans. Med. Imag., vol. 23, no. 11, pp. 1344-1352, Nov. 2004.

[31] H. Yoshida and J. Nappi, "Three dimensional computer aided diagnostic scheme for detection of colonic polyps," IEEE Trans. Med. Imag., vol. 20, no. 12, pp. 1261-1274, 2001.

[32] H. Yoshida, Y. Masutani, P. MacEneaney, D. Rubin, and A. H. Dachman, "Computerized detection of colonic polyps at CT colonography on the basis of volumetric features: Pilot study," Radiology, vol. 222, pp. 327-336, 2002.

[33] Walter Reed Army Medical Center Virtual Colonoscopy Center Collection Database [Online]. Available: http://virtualcolonoscopy. nci.nih.gov/main.html

[34] H. Frimmel, J. Nappi, and H. Yoshida, "Centerline-based colon segmentation for CT colonography," Med. Phys., vol. 32, pp. 2665-2672, 2005.

[35] P. F. Whelan, R. J. T. Sadleir, and O. Ghita, "NeatVision: Visual programming for computer-aided diagnostic applications," Radiographics, vol. 24, no. 6, pp. 1779-1789, 2004.
[36] R. J. T. Sadleir, P. F. Whelan, P. MacMathuna, and H. M. Fenlon, "Portable toolkit for providing straightforward access to medical image data," Radiographics, vol. 24, no. 4, pp. 1193-1202, 2004.

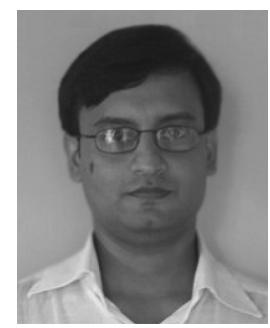

Tarik A. Chowdhury received the B.Eng. degree from the Rajshahi University of Engineering and Technology, Bangladesh in 1998 and the M.Eng. and Ph.D. degrees from Dublin City University (DCU), Dublin, Ireland, in 2003 and 2006, respectively.

$\mathrm{He}$ is currently an Assistant Professor in BRAC University, Dhaka, Bangladesh. He was a former member of the Vision Systems Group, DCU. His main research interests are in the area of image processing and medical imaging.

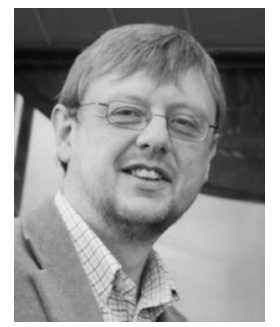

Paul F. Whelan (S'84-M'85-SM'01) received the B.Eng. degree from the University of Dublin, Dublin, Ireland, the M.Eng. degree from the University of Limerick, Limerick, Ireland, and the Ph.D. degree from the Cardiff University, Cardiff, U.K.

During the period 1985-1990 he was employed by ISI Ltd and later Westinghouse (WESL), where he was involved in the research and development of industrial vision systems. He was appointed to the School of Electronic Engineering, Dublin City University (DCU) in 1990 and is currently a Professor of computer vision (Personal Chair). He set up the Vision Systems Group in 1990 and currently serves as its Director. As well as publishing over 150 peer-reviewed papers, he has coauthored two monographs namely Intelligent Vision Systems for Industry (1997, Springer) and Machine Vision Algorithms in Java: Techniques and Implementation (2000, Springer). He has also coedited thee books including Selected Papers on Industrial Machine Vision Systems (1994, SPIE). His research interests include image segmentation and its associated quantitative analysis (specifically mathematical morphology, texture analysis) with applications in computer vision and medical imaging.

Dr. Whelan is a Chartered Engineer (IET). He serves on the governing board of the International Association for Pattern Recognition and is the current President of the Irish Pattern Recognition and Classification Society.

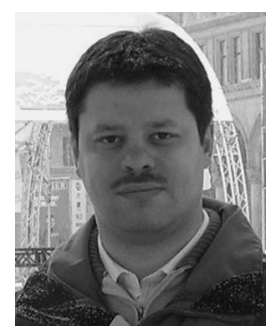

Ovidiu Ghita received the B.E. and M.E. degrees in electrical engineering from Transilvania University, Brasov, Romania and the Ph.D. degree from Dublin City University (DCU), Dublin, Ireland, in 2000, for work in the area of robotic vision.

From 1994 to 1996, he was an Assistant Lecturer in the Department of Electrical Engineering, Transilvania University. Since then he has been a member of the Vision Systems Group, DCU. Currently, he holds a position of Postdoctoral Research Assistant in the Vision Systems Group at DCU. He has authored over 65 peer-reviewed research papers in areas of instrumentation, range acquisition, machine vision, texture analysis, and medical imaging. 